analysis. Here, we aim to analyse risk preference in ADHD and healthy controls and the effects of MPH.

Methods Twenty-four healthy volunteers and 25 ADHD patients were tested on the 2 step sequential learning task in both MPH-ON and MPH-OFF conditions. We calculated the risk associated with each choice (variance of reward probability) and defined the choice with maximum variance as the risky one, for all 134 trials. With behavioural measures (selected choice- risky vs non-risky and response time) as inputs and risk as an independent factor, we extracted threshold $(a)$, drift rate $(v)$ and response bias $(z)$ parameters using a hierarchical drift diffusion model (HDDM) for both groups during $\mathrm{ON}$ and OFF drug condition. Statistical analysis on the parameters was analysed using Bayesian factors.

Results Bayesian repeated measures ANOVA showed evidence for changes in response bias $(z)$ but not in threshold and drift rate. A strong evidence for main effect of drug $\left(\mathrm{BF}_{10}=6.03 \times 10^{11}\right)$, group $\left(\mathrm{BF}_{10}=86344\right)$ and group by drug interaction $\left(\mathrm{BF}_{10}=3.65 \times 10^{6}\right)$ was observed. Post-hoc Bayesian independent sample t-tests showed strong evidence that the patient group had a higher preference towards the risky choice during both the $\mathrm{ON}\left(\mathrm{BF}_{10}=8.94 \times 10^{14}\right)$ and $\mathrm{OFF}$ $\left(\mathrm{BF}_{10}=20.9\right)$ conditions. Post-hoc Bayesian paired sample ttests showed strong evidence for the drug to induce a preference towards the risky choice in both the $\mathrm{HV}\left(\mathrm{BF}_{10}=397.1\right)$ and $\operatorname{ADHD}\left(\mathrm{BF}_{10}=1.16 \times 10^{10}\right)$ population. Behavioural results show a drug by group interaction $(F(1,0.01)=11.80, \mathrm{p}=0.001)$ on number of risky choices. Post-hoc analysis using paired sample t-test showed a significant increase in risky behaviour due to drug in the $\operatorname{ADHD}(t(24)=-3.5, \mathrm{p}<0.005)$ but not healthy subjects. No differences were found in the traditional reinforcement learning parameters between the groups.

Conclusions Using a novel analysis, we showed that ADHD subjects had a greater bias towards risk preference and further that $\mathrm{MPH}$ increases risk preference in both $\mathrm{ADHD}$ and $\mathrm{HV}$ with a comparatively greater effect on the patient population. Critically we observe an effect on response bias highlighting the role of apriori information in influencing risky decision making.

\section{STATE AND TRAIT INTEROCEPTION IS DISRUPTED IN FUNCTIONAL SEIZURES}

\footnotetext{
${ }^{1,2} \mathrm{M}$ Yogarajah, ${ }^{1} \mathrm{~A}$ Koreki, ${ }^{2,3} \mathrm{~N}$ Agrawal, ${ }^{3} \mathrm{~S}$ Cope, ${ }^{3} \mathrm{~T}$ Eilon, ${ }^{4,5} \mathrm{C}$ Gould Van Praag, ${ }^{5} \mathrm{~S}$ Garfkinel, ${ }^{5} \mathrm{H}$ Critchley, ${ }^{2,6} \mathrm{M}$ Mula, ${ }^{1,2} \mathrm{M}$ Edwards. 'Institute of Molecular and Clinical Sciences, Neurosciences Research Centre, St George's University of London; ${ }^{2}$ Atkinson Morley Regional Neuroscience Centre, St George's Hospital, London; ${ }^{3}$ Department of Neuropsychiatry, South West London and St George's Mental Health Trust, London; ${ }^{4}$ Department of Psychiatry, University of Oxford, Oxford; ${ }^{5}$ Brighton and Sussex Medical School and the Sackler Centre for Consciousness Science, University of Sussex; ${ }^{6}$ Institute of Medical and Biomedical Education, St George's University of London
}

\subsection{6/jnnp-2019-BNPA. 17}

Objectives The continuity and integrity of a conscious sense of self, is proposed to be dependent upon the control of internal physiological state and its predictive representation through interoception, that is, the sensing of internal bodily changes. We investigated dissociation, interoception and their relationship, in patients with functional seizures (FS), before and after a stressor intervention.

Methods 41 participants with functional seizures (FS) and 30 age/gender matched healthy controls (HC) were assessed with the somatoform dissociation questionnaire (SDQ20), multi-scale dissociation inventory (MDI), and the state and trait anxiety inventory (STAI). Standardized measures of interoceptive sensibility, accuracy, and awareness were acquired with the Porges Body Perception Questionnaire (PBPQ), and heartbeat discrimination (HDT), tracking (HTT) and timetracking tasks (TTT), before and after a cold pressor test. Continuous non-invasive blood pressure monitoring was carried out before, during and after the cold pressor test. Interoceptive trait (ITPE) and state (ISPE) prediction errors, that is, the discrepancy between interoceptive accuracy and the PBPQ (trait), and trial-by-trial confidence estimates (state), were calculated before and after the cold pressor test respectively, for HTT and HDT. An autonomic prediction error (APE), or the discrepancy between the reported increase in pain and the change in blood pressure after the cold pressor, was also calculated.

Results Patients with FS differ significantly from HC for HTT, ITPE and ISPE suggesting that they are overall less interoceptively accurate and aware than HC. This is confirmed by a correlation between APE and the ISPE derived from the HDT task $(r=0.359, p=0.033)$ in FS subjects only, after correcting for state anxiety and duration of cold pressor. Furthermore, in FS patients only, ITPE scores, adjusted for trait anxiety, correlated with SDQ-20 and MDI-depersonalization scores for both HTT $(r=0.378, p=0.008 ; r=0.408, p=0.005)$ and HDT $(\mathrm{r}=0.364, \mathrm{p}=0.011 ; \mathrm{r}=0.281, \mathrm{p}=0.044)$. All results survived FDR correction at a 0.05 threshold.

Conclusions These findings demonstrate that state and trait interoception are disrupted in patients with FS. The severity of the disruption in trait interoception correlates with measures of dissociation, such that the bigger the ITPE, the more severe are the dissociative traits. Similarly, the greater the ISPE, the larger the discrepancy between subjective symptoms and objective physiological changes, after a stressor intervention. Our findings suggest that the selective disruption of interoceptive processing is both a potential predisposing and precipitating factor in FS.

\section{Members' POSTER Abstracts}

\section{HIGH LEVELS OF ANXIETY AND DEPRESSION IN PATIENTS ATTENDING WITH HEADACHES TO A UK GENERAL NEUROLOGY CLINIC}

Thomas Cronin*, Ronald Pearce.

\subsection{6/jnnp-2019-BNPA. 18}

Objective The published literature on headache epidemiology comes from specialist headache clinics, compared to the general neurology clinic. This study set out to investigate the characteristics and diagnoses of patients with headaches attending a general neurology clinic in the UK.

Methods Data were collected retrospectively from a two-year period on 217 patients with headaches referred to a general neurology clinic at a UK district-general hospital seen by a single consultant. Clinic letters were reviewed, and information was inputted using a pre-formed Microsoft Excel spreadsheet. All data were anonymised, with no identifiable patient characteristics being recorded.

Results A total of 217 were seen in this period. The mean age was $42 \%$ and $72 \%$ were female. In $56 \%$ of cases, more than one diagnosis was made. The most frequent diagnosis 
was migraine (72\%), followed by tension-type headache (56\%). Dizziness and/or vertigo was a commonly reported coexisting symptom, with $24 \%$ reporting this. For $13 \% \quad(n=29)$ of cases, it was documented that time was taken out of work or studies due to headache symptoms. In 205 patients it was reported whether sleep was affected, with $70 \%(n=144)$ of cases indicating it was. For 195 cases, 80\% $(n=155)$ reported a normal appetite, $16 \%(\mathrm{n}=32)$ a decrease, and $4 \%(\mathrm{n}=8)$ an increase in appetite. In 195 patients, 65\% $(n=126)$ reported reduced energy levels. For 21\% $(n=46)$ there was documentation of anxiety. Regarding mood, in 176 cases where this was recorded, 33\% gave a negative mood description.

Conclusion To the authors' knowledge, this study is the first to report on headache characteristics in patients presenting to a UK general neurology clinic. The diagnostic frequency of different headaches presented in this study are comparable to those described in specialist headache clinics. Our population demonstrate significant psychiatric morbidity associated with headaches, with $33 \%$ reporting negative mood value and in addition higher proportions reporting energy and sleep disturbance. To conclude, this study has shown the common headache diagnoses encountered in a general neurology clinic, and indeed is comparable to headaches managed in primary care. Improved integration between these services is key to ensuring effective care for such patients.

\section{9 'NOBODY PREPARED ME FOR THIS!' PARENTS' EXPERIENCES OF SEEKING HELP AND SUPPORT WITH POST-BRAIN INJURY SYMPTOMS AND CHANGES IN CHILDREN AND ADOLESCENTS WITH ACQUIRED BRAIN INJURY}

Robyn MCCarron*

\subsection{6/jnnp-2019-BNPA.19}

Objectives/aims This research aims to:

- Understand the journeys and processes that parents follow in seeking help and support for post-brain injury symptoms and changes in their children.

- Identify the barriers and challenges that parents face in obtaining help and support for their children and family following paediatric Acquired Brain Injury (ABI).

- Propose ways in which services can be more responsive to the evolving needs of children and adolescents with ABI and their families.

Method Five mothers were interviewed using semi-structured interviews about their experiences of seeking help and support for post-brain injury symptoms and changes in their children. The participants' children were 60\% male, 10-16 years old and were 2-6 years post injury. Four had suffered strokes and one had a Traumatic Brain Injury. The age at injury was 4-14 years old. The interviews were audio-recorded and transcribed into 2698 units of data. The data was analysed using a Glaserian grounded theory constant comparative approach.

Results After a child suffers an ABI parents feel that it is down to them to ensure that their child gets the help and support that they need. Identifying and accessing support requires a high level of research and persistence that parents experience as a 'battle'. Obtaining support for mental-health problems is especially challenging. Common barriers include communication issues, time and funding delays, a lack of consistent professionals, not meeting the criteria for services, and a lack of local or age-appropriate services. The challenges of trying to seek help and support impact upon the whole family in terms of time, finances, socially and emotionally. However, through this process parents do become expert parents/carers. They gain new knowledge and language and they look to share this to support other parents going through similar experiences.

Conclusions Providing parents with accessible psychoeducation around post-brain injury symptoms and changes in the acute period following a paediatric ABI could be a helpful intervention to make parents feel more prepared and supported in the medium to long term. More specialist paediatric ABI services that can provide integrated, holistic and developmental care are needed. Current services may need a more flexible and longer-term approach to be responsive to the needs of young people with ABI.

\section{CLINICAL CHARACTERISTICS OF PATHOLOGICAL CONFIRMED EARLY ONSET DEMENTIA WITH LEWY BODIES}

1,2,3Simon Kang Seng Ting ${ }^{*},{ }^{1}$ Celeste Chen, ${ }^{4}$ Huihua Li $1,2,3$ Shahul Hameed, ${ }^{2}$ Adeline NG, 1,2,3 Eng-King Tan, ${ }^{2,5} \mathrm{Kok}$ Pin Ng, ${ }^{2,3}$ Nagaendran Kandiah. ${ }^{1}$ Department of Neurology, Singapore General Hospital, Singapore, Singapore; ${ }^{2}$ Department of Neurology, National Neuroscience Institute, Singapore, Singapore; ${ }^{3}$ Duke-NUS Medical School, Singapore; ${ }^{4}$ Health Services Research and Biostatistics Unit, Singapore General Hospital

\subsection{6/jnnp-2019-BNPA.20}

Background Early-onset dementia (EOD) is characterized by distinct clinical profiles and prognosis when compared to lateonset dementia (LOD). As the second most common neurodegenerative form of dementia, little is known about the clinical profile of early-onset Dementia with Lewy Bodies (DLB). A current challenge for clinicians when managing patients with DLB is the suboptimal diagnosis rate which will affect treatment efficacy and outcome. To address this knowledge gap, by hypothesizing early-onset DLB will have a distinct profile when compared to Alzheimer's disease (AD), we accessed and reviewed data of patients with pathological confirmed DLB from National Alzheimer's Coordinating Center (NACC) database.

Methods Patients with first visit that fulfill criteria for dementia of AD or DLB were analyzed. Early onset age was defined as less than 65 years old. Variables included in the analyses include baseline demographics, cognitive, behavioral, motor symptoms, neuropsychological battery scores and clinician diagnosis. Comparisons were made between early-onset $\mathrm{AD}$ (EOAD) versus early-onset DLB (EODLB), and early versus late-onset DLB.

Results This study included 363 patients with EOAD, 32 EODLB and 147 late-onset DLB. Patients with EODLB were more likely to present with psychosis, apathy, REM sleep behavioral disorder, and motor symptoms. While EOAD patients were more likely to present with cognitive symptoms as first recognized and predominant presentation and perform worse in memory assessment. Motor as first recognized presentation, slowness, visual hallucination, caregiver reporting of agitation and apathy were the significant predictors to differentiate the two. Late-onset DLB patients were less depressed and more impaired in memory and executive function related scores than EODLB. Significant number of EODLB patients were misdiagnosed as $\operatorname{EOAD}(46.9 \%, \mathrm{p}<0.0001)$. 